\title{
El Logos Vallejiano entre lo Dialéctico y lo Trílcico
}

Existen lagunas importantes en la valoración crìtica de César Vallejo, a pesar de la extensa bibliografía sobre su obra y su persona. La más notable es la ausencia en caulquier idioma de un estudio integrador de toda su obra, en poesia y prosa, ausencia que hasta ahora quizá se explique por la envergadura de tal labor, y, hasta los últimos años, por las ediciones y versiones poco fehacientes de su obra, la que, en algunos casos, todavía es de acceso difícil.

No tan explicable, dado el interés por Vallejo de la crítica izquierdista, es la carencia de un verdadero enfoque marxista que abarque la obra vallejiana en su totalidad. En cuanto a la deficiencia de la crïtica vallejiana en idioma inglés, quizá las dificultades de traducción que presenta su poesĭa la explican en parte. Pero todo proyecto de tal índole supone un estudio exhaustivo de su obra, el que no ha existido hasta ahora en ningún idioma en cuanto a su segundo libro, Trilce. No obstante el interés en poemas específicos, ningún crítico hasta ahora ha hecho un examen detallado de todos los poemas de este libro. A pesar de que la crítica vallejiana suele considerar a este poemario como una unidad poética, ha faltado también una demostración rigurosa de este presupuesto crítico.

Aunque el estudio integrador y total de la obra vallejiana queda por hacer, en el último año y medio Jean Franco nos ha dado el primer análisis interpretativo de Vallejo en inglés, Guillermo Alberto Arévalo ha querido darnos la primera lectura marxista en castellano, y Eduardo Neale-Silva ha llevado a cabo una enorme tarea exegética de Trilce. ${ }^{1}$ Los tres estudios representan los dos polos complementarios de la crítica vallejiana; por un lado, el polo integrador que busca relacionar el poeta y su obra al mundo socio-político-cultural 
de que forma parte, o sea la crítica del escritor y su obra en el mundo - es el propósito de los libros de Franco y Arévalo; por otro lado, el polo analítico que se ciñe a los textos de Vallejo en busca de los significados más profundos de su expresión hermética, es decir, la critica del mundo del escritor en su obra- es el propósito del libro de Neale-Silva.

Al hacer un enfoque fundamentalmente textual, Neale-Silva ha evitado precisamente el estudio conceptual que supone un profundo conocimiento anterior del libro. Puesto que cree que "no es legítimo hacer estudios conceptuales sobre el arte trilcico antes de analizarlo y entenderlo", se ha dedicado principalmente a esta labor, "sabiendo que tal tarea no podrá hacerse jamás en forma exhaustiva" (p. 23). Sin embargo, cabe preguntarse si será superada jamás la labor monumental que representa este estudio. El autor ha buscado integrar los datos y juicios más importantes de la crìtica vallejiana hasta la fecha de 1972 o 1973, año en que quedó terminado el libro (aunque no se publicó hasta más de cuatro años después), y asimilarlos a sus propias investigaciones y juicios sobre los poemas de Trilce.

El estudio se divide en dos partes. La primera, de más extensión, trata de los conceptos y vivencias de la vida intelectual y emocional del poeta Vallejo (a quien el crítico distingue del hombre Vallejo); preocupaciones político-sociales, sicológicas, filosóficas, aspectos vitales de las vivencias del poeta tales como su niñez, la figura de la madre, el valor de la familia, el traumatismo psíquico del encarcelamiento, las experiencias amorosas, la pérdida del hogar y la bohemia limeña. La segunda parte analiza los distintos "modos de significación": funciones simbólicas, forma interior, técnicas estilisticas como el prosaismo intencional y puerilización poética, la ambigüedad, el desdoblamiento lírico, la desarticulación onirica, más el papel del pitagorismo y de los números. Se trata, pues, de dos énfasis distintos, el primero sobre el contenido poético, el segundo sobre los modos de significación, sin que se excluyan el uno al otro para desembocar en una falsa concepción bipartita del arte poético.

A lo largo de su análisis Neale-Silva ha seguido dos procesos "que se suceden dentro de un orden fijo: 1) comprensión de los significados primordiales y secundarios de cada poema y 2) elaboración de panoramas valorativos sobre fundamentos genéricos propios de Trilce y de Vallejo en general" (pp. 22-23). O sea, va siempre del análisis a la sǐntesis, y siempre a base de la tesis de que no es legútimo hacer estudios conceptuales sobre el arte trilcico antes de analizarlo y entenderlo. 
A pesar de esta estructuración general, la metodología que emplea el profesor Neale-Silva es declaradamente ecléctica: explicitaciones hermenéuticas, análisis lógico, aproximaciones estilî̀sticas, estudio gramatical, semántico y lexicográfico del vehículo poético, enfoques fenomenológicos, elementos de historia literaria y valoraciones estéticas. Además, los aspectos que estudia Neale-Silva no representan un inventario exhaustivo de los temas posibles, como lo indica el propio crítico (en un apéndice señala casi cincuenta temas relacionados que pudieran ser tratados en el examen crítico de Trilce), ni expone explïcitamente por qué ha escogido estos temas y no otros. Tampoco ha buscado dar una visión de conjunto de la materia poética (valores, actitudes, intuiciones, carga emocional, motivos, ideaciones, etc.), ni de la configuración y expresión, ni de la fundamentación teórica del poemario.

Es ésta, por eso, la base de otro estudio por realizar, precisamente el de la visión de conjunto. Pero el hecho de ser, por decirlo asǐ, un estudio preliminar, no resta ningún valor a este libro único. En primer lugar, los análisis, aunque no pretenden ser definitivos, casi siempre convencen como lecturas posibles, y aun cuando no del todo, es muy raro que el lector no aprenda algo nuevo sobre el poema que le permite acercarse más a los significados que se le asoman en segundas lecturas. El propio crítico señala el hecho de que "tratándose de un poeta como Vallejo, es imposible dar opiniones absolutas" (p. 13). Por eso, al discrepar de los análisis de otros críticos, puede hacerlo con una generosidad ejemplar, puesto que no tiene ninguna causa ideológica que defender. Así se salva de otro naufragio posible: las polémicas literarias, políticas y personales que tanto caracterizan a mucha crítica vallejiana, y esto facilita el enfoque sin prejuicios de los poemas mismos.

He aquí el mayor valor crítico de la labor de Neale-Silva: el haber analizado los setenta y siete poemas de Trilce sin prejuicios que le impidan al crítico permanecer abierto a distintos valores poéticos e ideológicos. Sin duda tal monumento de labor erudita queda destinado a convertirse en una clásica obra de consulta. Además de ser una fuente asombrosa de datos bibliográficos y generales sobre la obra de Vallejo, las valoraciones pueden servir de puntos de partida para el lector, quien discrepe o no con la interpretación del crítico, tendrá que cumplir otro propósito que el de éste: el de despertar más interés en la obra de Vallejo.

Además de las interpretaciones específicas, Neale-Silva establece ciertas caracterǐsticas de Trilce que ayudan al lector a adentrarse más 
en esta poesǐa: la potencialidad semántica que se presta a distintas interpretaciones posibles, y que se deriva de la ambigüedad deliberadamente buscada (pp. 426-427), las calidades plásticas de Trilce (p. 496 y 496 n.), la unidad de las composiciones trílcicas (p. 471), aspectos básicos del simbolismo trílcico, temas o motivos que se repiten, y los distintos niveles lingüisticos y estilísticos.

Sin embargo, el crítico no nos da un concepto total del poeta Vallejo por no incluir su estudio tal finalidad entre sus propósitos. Lo que sỉ nos da, más de un medio siglo y muchas páginas de crǐtica literaria después de la publicación de Trilce en 1922, es la fundamentación pormenorizada de una comprensión más profunda del poeta durante su fase trilcica, fundamentación imprescindible para llegar a un concepto más adecuado del poeta.

Tal concepto total del poeta nos lo ofrece Jean Franco en su estudio, el cual, ya en su título, destaca la dialéctica entre la poesía y el silencio, o sea, la lucha del poeta para encontrar un lenguaje capaz de expresar su mundo poético, aspecto fundamental del libro de Franco que es a la vez mérito y defecto. El mero hecho de ser el primer estudio global de Vallejo publicado en inglés, ya de por sỉ le otorga al libro una gran importancia. Además, el enfoque de Franco es penetrante, provocador, a veces discutible, pero con frecuencia brillante. Es un estudio sintetizador, orgánico, una tentativa de trazar la trayectoria de la producción de Vallejo a través de sus obras principales, Los heraldos negros (1918), Trilce (1922), Poemas en prosa (1923-1931), Poemas humanos (1931-37) y España, aparta de mi este cáliz (1937).

Franco centra su análisis en el desarrollo del lenguaje poético de Vallejo desde el romántico enajenado de su primer poemario y su descubrimiento de la "elasticidad" del lenguaje, hasta el reconocimiento, agudo y crucial en Trilce, de la contingencia de la identidad personal y la imposibilidad de expresar el logos o la verdad primordial, con el resultado concomitante de la conciencia falsa y la desmistificación de la poesía. Los poemas en prosa que escribió Vallejo desde su llegada en Parïs en 1923 hasta el año 1931 siguen ilustrando la importancia de la analogía de la pérdida de la identidad personal con el fracaso del lenguaje y del logocentrismo en Trilce. Pero tal crisis de conciencia iba a desarrollarse aún más a través de los años veinte hasta llevar a Vallejo a aceptar el marxismo como solución política, y a escribir, como solución poética, una descripción del yo atrapado en una prisión de su propia fabricación. Puesto que es un ser programado biológicamente por su naturaleza, el hombre puede 
pensar sólo dentro de una estructura dictada por la sociedad. De esta manera está esclavizado biológicamente y se encuentra disminuido a un papel meramente funcional dentro de un ambiente que ya no puede controlar.

Se presentan en Poemas humanos dos alternativas: una la de la utopía que revela el proceso por medio del cual el hombre puede estructurar y así cambiar su realidad, y otra, la del apocalipsis que revela a la humanidad atrapada por su propia historia y condenada a sí misma, si no da el sal to que la saque de su trayectoria individualista y destructora.

Los Poemas humanos, según el juicio de Franco, no son el producto de una estética definitiva, sino que representan una serie de exploraciones. Por eso, el lector debe tener en cuenta la "intertextualidad" de los poemas de esta época, puesto que representan un vaivén meditativo continuo sobre el destino del individuo. Compenetrado con la tristeza de los años treinta, la poesía de Vallejo revela una búsqueda continua del logos, aunque su poesía no es cristiana en modo alguno. Ni la escritura ni el habla reemplaza al logos, porque en el centro del discurso hay un vacio nacido de la incapacidad del lenguaje de expresar de inmediato la conciencia.

Vallejo señala constantemente el abismo entre el discurso y la materia prima de la existencia, por el cual el acto de escribir resulta enajenador. El lenguaje no es nada más que la suma de los significados humanos acumulados a través de la historia, y resulta ser no otra cosa que una estructura más para esconder la verdad, como la filosofía o la literatura, construída por la conciencia humana. El hombre, quien en el alba del tiempo histórico se declaró un ser aparte de la naturaleza, único, que produce por naturaleza, ahora encuentra superfluo todo lo que produce, incluso la poesía misma, la que, sin embargo, es un esfuerzo para negar su papel como productor de lo superfluo y de esa manera diferir su aniquilación.

Sólo en España, aparta de mí este cáliz encontramos el reconocimiento de un nuevo espíritu de la humanidad y de un nuevo verbo, productos del sacrificio de la Guerra Civil. Para Franco, este grupo de 15 poemas funde el espíritu mesiánico del Antiguo Testamento con el mensaje del Nuevo, el de que el hombre puede salvarse de la muerte. España viene a ser el texto en el que la salvación del hombre va siendo escrita por la acción colectiva de un pueblo que ha suprimido los egos individuales, destructivos, en nombre de la lucha por la libertad. Tal espíritu colectivo significa para Vallejo un salto cualitativo capaz de superar el individualismo egoỉsta, un salto que 
conducirǐa a una utopìa capaz de redimir al mundo entero, y este hecho le resultó tan importante que con él pretendió Vallejo justificar la carnicería en nombre de una causa buena distinta, por tanto, de otros tipos de matanzas.

En su lectura de Vallejo, Franco busca destruir el "Vallejo inventado", el que predijo su propia muerte: "Me moriré en Parìs con aguacero,/ un día del cual tengo ya el recuerdo./" Esta celebración de la personalidad del poeta, repugnante al propio poeta como lo evidencia su poesía, se desarrolló en una leyenda que se ha puesto como valla entre los lectores y la poesǐa de Vallejo. Mejor dicho, se desarro11 ó en leyendas, porque habìa varias. La publicación póstuma de Poemas humanos, con su sugerente título, vino a animar a los que emprendieron la crítica identikit y buscaron por debajo de las palabras al hombre, como si los poemas fueran los órganos de transplante en un trabajo de resurrección literaria que tuviera como único propósito el del redescubrimiento del Vallejo auténtico. Franco critica severamente a Juan Larrea, quien en su libro César Vallejo o Hispanoamérica en la cruz de su razón, convirtió a Vallejo en una figura mesiánica del mundo hispánico, martirizado por la inhumanidad del hombre para con su prójimo.

El problema que presentan todos estos mitos, afirma Franco, es que nos permiten no leer la poesia, y no enfrentarnos asỉ con la traición y la incompetencia del lenguaje, para poder de esa manera reconocer la diferencia entre lo inflexible y necesario y lo que podemos cambiar. Según Franco, el gran logro de Vallejo es el haber creado con cada poema un acto de conciencia que registra las dificultades de la vida y la existencia del dolor. Tal logro se debe al reconocimiento por parte del poeta de que cada palabra o gesto automático del hombre contribuye a su propia condenación y encarcelamiento. Por eso, los poemas no exigen que hagamos de Vallejo una víctuma propiciatoria para librarnos de la experiencia de la vida humana, sino que aceptemos las exigencias del texto.

Es un juicio acertado, aunque uno puede discrepar de las lecturas específicas de los poemas, y hasta con la tesis general de que Trilce representa la pérdida de la identidad individual o el rechazo por superflua de tal identidad. Hay cierta confusión, además, entre los términos individualismo y conciencia, confusión que hace a ambos conceptos equivalentes. Para Vallejo, según Franco, la conciencia humana se caracteriza por la malicia y parece ser "una energía sobrante y demoniaca" (p. 162), y a su turno forma parte de la antinomia conciencia/abnegación (consciousness/self-forgetfulness). 
Este último término representa la perfección, lo que quiere decir que "tenemos que aceptar la destrucción de la conciencia" (p. 163). Hay aquí, por lo menos, dos problemas. El más obvio es que más tarde Franco habla del desarrollo de la conciencia colectiva como la solución de Vallejo para la salvación del hombre. No es mera sutileza pedir un esclarecimiento de la distinción entre la conciencia colectiva y la individual. En segundo lugar, no es tan obvio que la conciencia individual sea idéntica al individualismo egoísta. Por eso, lo que puede señalarse como una conciencia de sî (self-consciousness) intensa en Trilce, la que Franco parece reconocer como la preocupación de Vallejo por el mecanismo por el que el yo poético y la "ilusión" de una psique individual se producen, no lleva consigo inevitablemente un egoǐsmo. Queda por demostrar también de una manera mucho más convincente cómo la humanidad puede llegar a la conciencia colectiva sin que primero los seres humanos tengan conciencia de sí mismos como individuos y luego como seres humanos de necesidades e intereses comunes.

Como queda señalado, las interpretaciones de poemas especificos no siempre convencen; con frecuencia Franco parece forzar el sentido para ajustarlo a su tesis. De la misma manera, puesto que se trata de traducciones, las versiones inglesas a veces parecen arbitrarias (no existe todavia, aparte de algunos poemas sueltos, una versión inglesa de la obra poética de Vallejo que sea digna de confianza), y dan, en su totalidad, la impresión de ser parte de una obra algo programada, puesto que pierden mucho de la unicidad que poseen como poemas individuales en el original castellano, sobre todo por la pérdida de mucha de su ambigüedad polisémica. Este estudio presupone la existencia de versiones inglesas adecuadas; para los lectores sin un conocimiento del castellano, no hay manera de poner en su contexto los poemas o fragmentos de poemas citados por Franco.

Sin embargo, la tesis de Franco logra dar coherencia a la figura de Vallejo, a la vez que ilumina toda una época. Evita la fragmentación de la obra poética de Vallejo al mostrar el hilo dialéctico que corre a lo largo de su trayectoria poética, las tensiones entre lo biológico y lo cultural, entre la naturaleza y el lenguaje, entre el yo y la colectividad social. Además, logra relacionar de esta manera la poesía con el mundo de Vallejo como hombre, evitando el quedarse atrapado en una esterilidad formalista que elimine toda dialéctica entre la realidad poética y la realidad extraliteraria, y a la vez sin forzar artificiosamente tales relaciones entre estas dos realidades.

El estudio de Guillermo Alberto Arévalo no llega a tal coheren- 
cia. Por su título, César Vallejo/Poesia en la historia, demuestra el deseo de insertar la poesia de Vallejo en el mundo del hombre Vallejo, mejor dicho, en el mundo de todos los hombres. Arévalo encuentra el rasgo caracterîstico de la poesỉa de Vallejo en su ruptura con la tradición, "el desgarrón vallejiano", y la causa histórica de este desgarrón vallejiano, reflejado en la obra, es el tema fundamental de su estudio. Hay cierta semejanza entre la tesis de Arévalo y la de Franco, pero expresada en distintos términos y con menos destreza. No demuestra Arévalo la misma capacidad sintetizadora que Franco, quien evidencia una vasta erudición y logra dar un perfil conceptual a todo el siglo pasado de la cultura occidental. Por eso, quizá, no nos convence Arévalo con su teoría marxista del materialismo histórico que busca plantear científicamente un origen histórico del arte. Y no es que no nos convenza en sî tal teoría; la mera existencia de España, aparta de mi este cáliz comprueba la verdad que encierra.

Pero Arévalo parece confundir su método crítico con la praxis poética de Vallejo; o sea, en vez de demostrar las relaciones más profundas entre la poesía y la vida de Vallejo, Arévalo cede a la tentación de demostrar que Vallejo es marxista ortodoxo a lo largo de su trayectoria poética, para poder rechazar así a los críticos como Juan Larrea que quieren hacer de Vallejo un mistico contemporáneo - Arévalo tiene el mismo propósito desmistificador de Jean Franco. Por eso Arévalo pretende encontrar en un poema como "La cena miserable", de Los heraldos negros, una crítica al capitalismo explotador y al imperialismo internacional. La preocupación social de Vallejo no queda en duda; lo que no podemos aceptar es que la poesía de Vallejo de cualquier época de su vida demuestre tan superficialmente cierta crítica política. El bolchevique de "Salutación angélica" (Poemas humanos) asume contornos ideales, pero Vallejo nunca llega a predicar una ortodoxia marxista; queda su prosa de los años treinta -la novela Tungsteno, por ejemplo- para tal propósito propagandista.

Sin embargo, Arévalo ha sabido apuntar hacia un aspecto todavía no bastante estudiado en la obra de Vallejo: el de la relación entre el desarrollo intelectual y político del hombre y su expresión poética, sobre todo en cuanto al impacto del concepto del materialismo dialéctico en su obra total, tanto en su prosa como en su poesía. Según Arévalo, "el marxismo de Vallejo no sólo reside en sus obras de denuncia y militancia, sino que impregna hasta los más personales problemas", incluso la lírica de Poemas humanos (p. 138). Si se habla 
más especỉficamente del materialismo dialéctico, podemos aceptar este juicio, el cual equivale a decir que su poesía asume dimensiones políticas sólo en el sentido expresado por Vallejo mismo, cuando señala que toda expresión humana, por ser humana, tiene valor político en el sentido más amplio. Pero no por eso es literatura puesta al servicio de un partido o sometido a una consigna. El Vallejo prosista de cierta época, la de los años treinta, se puso al servicio del Partido Comunista, pero su poesỉa nunca llegó a ser un instrumento de propaganda. Sin embargo, Vallejo no quedó inafectado por sus estudios marxistas. Establecer los nexos más secretos de esta acción, y configurar asĭ más precisamente la expresión poética máxima de Vallejo, la de Poemas humanos y España, aparta de mí este cáliz, queda como otra tarea más que debe realizar la crítica vallejiana.

University of Pittsburgh

KEITH A. MCDUFFIE

\section{NOTA}

1. Jean Franco, César Vallejo: The Dialectics of Poetry and Silence (New York: Cambridge University Press, 1976); Guillermo Alberto Arévalo, César Vallejo/Poesia en la historia (Colombia: Carlos Valencia Editores, 1977); Eduardo Neale-Silva, César Vallejo en su fase trílcica (Madison: University of Wisconsin Press, 1976). 
